\title{
miR-339-5p inhibits lung adenocarcinoma invasion and migration by directly targeting BCL6
}

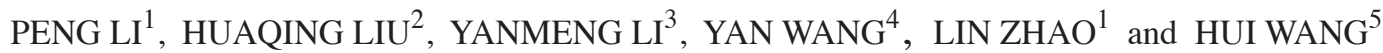 \\ ${ }^{1}$ Department of Respiratory Medicine, People's Hospital of Rizhao, Rizhao, Shandong 276800; \\ ${ }^{2}$ The Third Department of Neurology, Zhangqiu People's Hospital, Jinan, Shandong 250200; \\ ${ }^{3}$ Department of Laboratory Medicine, People's Hospital of Rizhao, Rizhao, Shandong 276800; \\ ${ }^{4}$ Department of Public Health, Zhangqiu People's Hospital, Jinan, Shandong 250200; \\ ${ }^{5}$ The Second Department of Respiratory Medicine, People's Hospital of Linyi, Linyi, Shandong 276003, P.R. China
}

Received March 2, 2018; Accepted August 8, 2018

DOI: $10.3892 / \mathrm{ol} .2018 .9376$

\begin{abstract}
Lung adenocarcinoma (LA) is a common non-small cell lung cancer, but effective biomarkers to diagnose early LA are still lacking. Increasing evidence has indicated that the dysregulation of microRNAs (miRNAs) play crucial roles in LA progression. miR-339-5p has been recently confirmed to exert crucial functions in various cancers. Nevertheless, molecular mechanisms and effects of miR-339-5p on LA development still remain vague. In the present research, miR-339-5p expression was downregulated in human LA tissues. miR-339-5p overexpression in LA cells could remarkably suppress the LA cell invasion and migration. In addition, further studies indicated that miR-339-5p overexpression downregulated both the B-cell lymphoma 6 (BCL6) mRNA and protein expressions by targeting the BCL6 3'-UTR directly. Moreover, BCL6 knockdown could partially lessen the function of miR-339-5p in LA invasion and migration. In conclusion, the present data identified miR-339-5p as a novel LA suppressor which exerted its functions partly by negatively regulating BCL6.
\end{abstract}

\section{Introduction}

Lung adenocarcinoma (LA), accounting for about half of lung cancers, is one of the most common non-squamous cell tumors (1). As one major type of tumor-related death globally, lung cancers are usually diagnosed at advanced stage which is not the optimal period for treatment (2). Although the lung cancer survival rate has increased due to the improved diagnosis and treatments, lung cancer overall prognosis is still poor and there is an urgent need for new therapeutic strategies for lung

Correspondence to: Dr Hui Wang, The Second Department of Respiratory Medicine, People's Hospital of Linyi, 27 Eastern Jiefang Road, Lanshan, Linyi, Shandong 276003, P.R. China

E-mail:wr83ww@163.com

Key words: lung adenocarcinoma, invasion, migration, miR-339-5p, B-cell lymphoma 6 cancer patients (3). Moreover, the molecular mechanism of LA remains to be fully elucidated (4). Therefore, understanding the molecular mechanism of LA is important to identify valid therapeutic biomarkers and develop LA treatment strategies.

MicroRNAs (miRNAs) are promising molecular biomarkers for the treatment of LA (5). miRNAs are a series of small non-coding RNAs made up of 18-25 nucleotides which can induce the inhibition of their target genes by binding to their 3'-UTRs and the suppression of translation (6). miRNA is one crucial factor in the progression and tumorigenesis of multiple tumors, and is also considered as potential tumor suppressor or oncogene, depending on the specific target gene (7). For instance, Yang et al found that miR-203 could suppress gastric cancer cell proliferation, invasion and migration via regulating Slug (8). Feng et al reported that miR-556-3p promoted human bladder cancer cell proliferation, migration and invasion by negatively regulating DAB2IP (9). miR-4317 was found to suppress human gastric cancer cell proliferation by targeting ZNF322 (10). Aberrant miRNA expression has been observed in lung cancer, such as downregulation of miR-146-5p (11) and upregulation of miR-616 (12). However, the biological effects of miR-339-5p on LA pathophysiological progression remain to be established.

B-cell lymphoma 6 (BCL6), one of the zinc-finger transcription factors, is selectively expressed by $\mathrm{T}$ and $\mathrm{B}$ cells within GCs (13). BCL-6 is a transcriptional repressor and proto-oncogene, contributing to the control of differentiation and cell cycle, as well as the inhibition of apoptosis (14). Tumor metastasis and invasion are dynamic, multistep and complex processes which are the major factors of tumor-related deaths. (15). Previous studies have demonstrated that BCL6 has a crucial effect on cell metastasis and invasion in breast cancer (16). In addition, BCL6 is regarded as a therapeutic target for autoimmune diseases as well as tumor treatment (17). Nevertheless, the expression and function of BCL6 in LA remain largely unclear. In this study, we measured BCL6 expression and investigated the correlations between miR-339-3p and BCL6 in LA.

\section{Materials and methods}

Patient specimens. Paired human LA specimens and adjacent normal tissue specimens were obtained from 50 patients 
who received surgical resection between May 2016 and January 2018 at the People's Hospital of Rizhao (Rizhao, China). All surgical samples were snap-frozen in liquid nitrogen and stored at $-80^{\circ} \mathrm{C}$ following resection until RNA extraction was performed. None of the patients received chemotherapy or radiotherapy prior to surgery. Written informed consent from each patient was obtained prior to the enrolment in the current research, which was approved by the Ethics Committee of the People's Hospital of Rizhao.

Cell lines. The LA A549 cells and the normal lung epithelial cells BEAS-2B were purchased from the Cell Bank of China Academy of Sciences (Shanghai, China) and maintained in RPMI-1640 medium (Invitrogen; Thermo Fisher Scientific, Inc., Waltham, MA, USA) containing 10\% FBS. All the cell lines were cultured in a humidified atmosphere of $5 \% \mathrm{CO}_{2}$ at $37^{\circ} \mathrm{C}$.

Cell transfection. miR-339-5p mimics, inhibitor or BCL6 siRNA as well as the corresponding controls were obtained from Shanghai GenePharma Co., Ltd. (Shanghai, China) and transfected into LA cell line A549 by Lipofectamine ${ }^{\circledR} 2000$ (Invitrogen; Thermo Fisher Scientific, Inc.) following the manufacturer's instructions.

Reverse transcription-quantitative PCR (RT-qPCR). TRIzol reagent (Invitrogen; Thermo Fisher Scientific, Inc.) was used to extract total RNA from LA tissues and cells. PrimeScript ${ }^{\mathrm{TM}}$ RT reagent kit (Takara Biotechnology Co., Ltd., Dalian, China) was used to reverse transcribe RNA into complementary DNA (cDNA). The amplifications of cDNA were conducted with the SYBR-Green Master Mix kit (Takara Bio, Inc., Otsu, Japan) in strict accordance with the manufacturer's guidelines on the system of ABI 7900 Sequence Detection System (Applied Biosystems; Thermo Fisher Scientific, Inc., Foster City, CA, USA). The expression of miR-339-5p and BCL6 was normalized to U6 and GAPDH, respectively. The relative gene expression was evaluated by the $2^{-\Delta \Delta \mathrm{Cq}}$ method (18).

Western blot analysis. Whole cell lysates were obtained from LA cells using ice-cold RIPA lysis buffer which contained protease inhibitor cocktail (Roche Diagnostics, Basel, Switzerland). A BCA protein assay kit (Beyotime Institute of Biotechnology, Haimen, China) was used to determine the protein concentrations. Then, the proteins were separated on SDS-PAGE and transferred onto PVDF membranes (Whatman International Ltd., Maidstone, UK). The membrane was blocked in 5\% fat-free milk for $2 \mathrm{~h}$ and treated with the diluted primary antibodies at $4^{\circ} \mathrm{C}$ overnight: A rabbit antibody against BCL6 (1:5,000; cat. no. ab19011) and a rabbit antibody against GAPDH (1:3,000; cat. no. ab9485) both from Abcam (Cambridge, UK). The membrane was washed with TBST 3 times and then was incubated with horse-radish peroxidase-linked secondary goat anti-rabbit antibody (1:4,000; cat. no. ab6721; Abcam). The western blot analysis results were analyzed using the enhanced chemiluminescence system (Pierce; Thermo Fisher Scientific, Inc.).

Transwell assays. The invasion and migration of transfected cells were determined by Transwell assay. Transwell chambers (Costar, Cambridge, MA, USA) were coated with Matrigel on the upper surface for invasion assay or without Matrigel for migration assay. For tumor cell invasion analysis, after incubation for $30 \mathrm{~min}$ at $37^{\circ} \mathrm{C}$, the solidified Matrigel was used as the extracellular matrix. Then, the LA cells were resuspended in serum-free medium in the top chamber, when the lower chamber was added with medium containing $10 \%$ FBS. After the incubation for $48 \mathrm{~h}$ at $37^{\circ} \mathrm{C}$, the cells in top chambers were removed carefully with cotton swabs, followed by fixing and staining the cells adhering to the lower surface for detecting the results. Additionally, the migration analysis was the same as the invasion assay except the Matrigel. The invasion or migration numbers of LA cells were counted with an inverted microscope (Olympus Corp., Tokyo, Japan) from five randomly selected visual fields.

Luciferase reporter assay. The Dual-Luciferase Reporter Assay System (Promega Corp., Madison, WI, USA) was used to confirm the precise target of miR-339-5p. The amplified BCL6-3'-UTR-WT or corresponding BCL6-3'-UTR-MUT was, respectively, inserted into pGL3 luciferase vector. LA cells were seeded into 24-well plates and incubated for $24 \mathrm{~h}$. Subsequently, the cells were co-transfected with luciferase reporter vectors of the wild-type or mutant type 3'-UTR of BCL6 gene and miR-339-5p mimics using Lipofectamine ${ }^{\circledR} 2000$ (Invitrogen; Thermo Fisher Scientific, Inc.). Moreover, the relative luciferase activities were detected with the Dual-Luciferase Reporter Assay kit (Promega Corp.) $48 \mathrm{~h}$ after the transfection.

Statistical analysis. The above experiments were performed 3 times. The statistical analysis was evaluated by the GraphPad Prism 6 (GraphPad Software, Inc., La Jolla, CA, USA) together with SPSS 18.0 version (SPSS, Inc., Chicago, IL, USA). Student's t-test was applied to evaluate the differences between the two groups. The data are indicated as means \pm SD. We used the Spearman's correlation analysis to determnine the correlation between miR-339-5p and BCL6 expressions in LA tissues. The differences were identified as statistically significant at $\mathrm{P}<0.05$.

\section{Results}

miR-339-5p expression is reduced and BCL6 expression is increased in $L A$. In the present study, we determined the miR-339-5p expression in 50 LA tissue specimens and normal tissues using RT-qPCR. Results indicated that, compared with the control group, the miR-339-5p expression in LA tissues were increased significantly (Fig. 1A). Additionally, we also measured the miR-339-5p expressions in LA cell lines. The results demonstrated a significant decrease of miR-339-5p expression in LA cells (Fig. 1B). Furthermore, we also measured the BCL6 expression in the LA cells. From the results, we knew that compared with the control group, the BCL6 expressions in the LA cells were notably enhanced (Fig. 1C). Next we analyzed the Spearman's correlation between miR-339-5p and BCL6 expressions in LA tissues. Based on the analysis results, the miR-339-5p expression was inversely related to the BCL6 expression in LA tissues (Fig. 1D). 

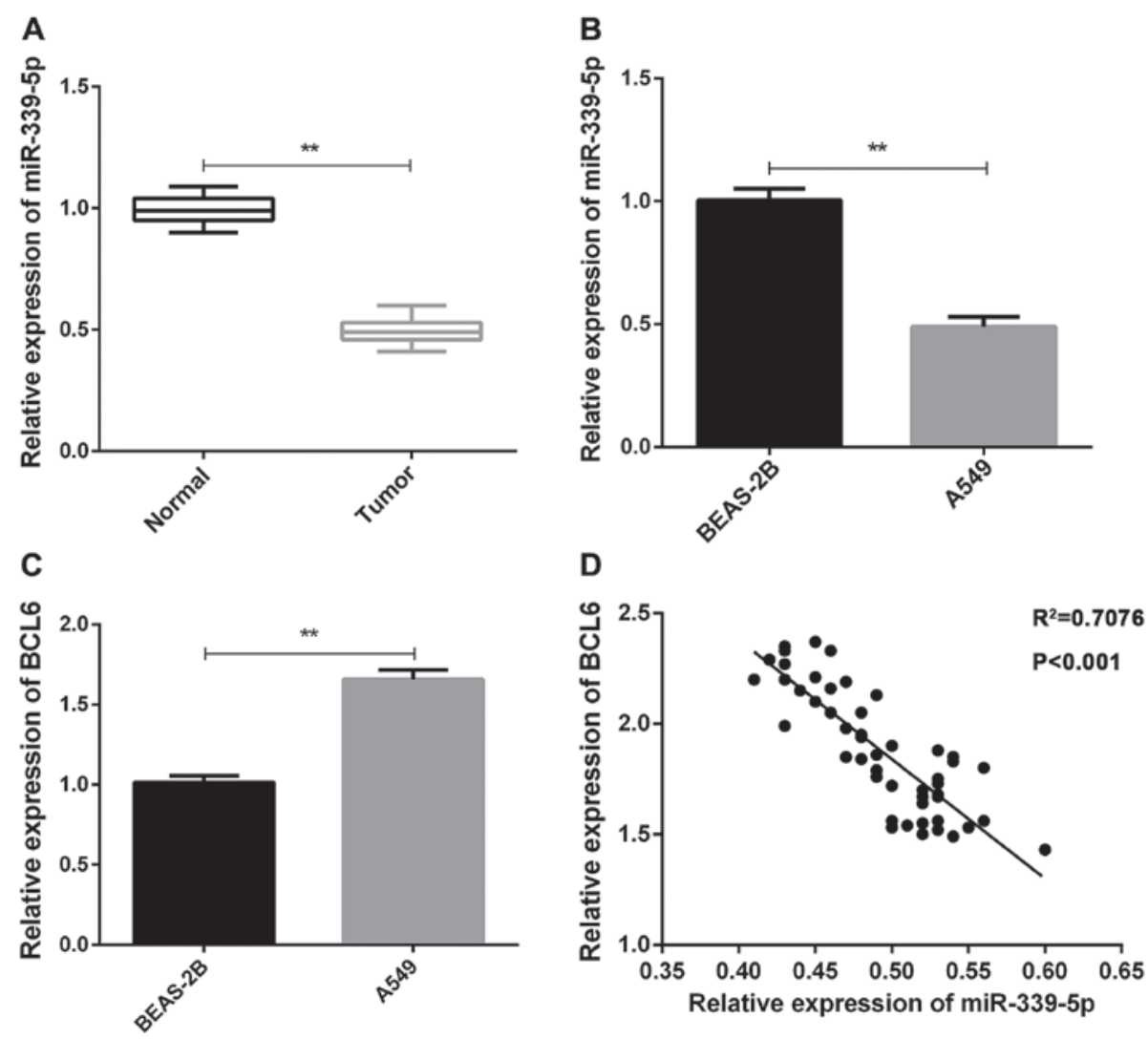

Figure 1. Analysis of expression of miR-339-5p and BCL6 in LA tissues and cells. (A and B) The expression of miR-339-5p in LA tissues and cells was measured using RT-qPCR ("* P<0.01). (C) The BCL6 expression in LA cells was evaluated by RT-qPCR (** P $<0.01)$. (D) Spearman's correlation between miR-339-5p and BCL6. BCL6, B-cell lymphoma 6; LA, lung adenocarcinoma; RT-qPCR, reverse transcription-quantitative PCR.

Effects of miR-339-5p on LA cell invasion and migration. To better understand the effects of miR-339-5p on LA cell invasion and migration, miR-339-5p mimics or inhibitor was transfected into a common LA cell line A549. Briefly, miR-339-5p mimics or inhibitor was transfected into A549 cells to up- or downregulate miR-339-5p expression (Fig. 2A). Then, we performed Transwell assays to investigate the mechanism of miR-339-5p in LA invasion. As shown in Fig. 2B and D, miR-339-5p restoration in LA cells resulted in declined cell invasion, whereas miR-339-5p silence in LA cells notably increased LA invasion compared with the control group. Subsequently, we continued to investigate the functions of miR-339-5p in LA cell migration. Data of Transwell assays also demonstrated that miR-339-5p overexpression remarkably repressed the LA cell migration and conversely, inhibition of miR-339-5p in LA cells promoted the capacity of LA cells (Fig. 2C and E).

miR-339-5p directly targeted BCL6 in LA cells. To investigate the underlying mechanism of miR-339-5p in LA, TargetScan was used to identify the potential target genes for miR-339-5p involved in LA progression. Based on the results, BCL6 was chosen as a candidate target for miR-339-5p. The putative wild-type or mutant BCL6 3'-UTR was inserted into luciferase reporter gene to create BCL6 3'-UTR-WT and BCL6 3'-UTR-MUT (Fig. 3A). Then, the LA cells were co-transfected with the corresponding vector containing wild-type/mutant BCL6 3'-UTR and miR-339-5p mimics to perform the luciferase reporter assays. The results demonstrated that the BCL6 3'-UTR-WT luciferase activity was notably decreased by
miR-339-5p mimics, whereas it had no influence on the BCL6 3'-UTR-MUT luciferase activities (Fig. 3B). Additionally, we found that miR-339-5p could modulate the expression of BCL6 mRNA and protein by RT-qPCR and western blot analysis. Briefly, the results indicated that miR-339-5p restoration in LA cells leads to downregulation of BCL6 expression and miR-339-5p inhibition contributed to upregulation of BCL6 expression (Fig. 3C and D). The data revealed that miR-339-5p regulated BCL6 expression at both mRNA and protein level via targeting its 3 '-UTR directly.

miR-339-5p triggers the inhibition of LA cell invasion and migration partially by regulating $B C L 6$. To further investigate whether the repression of LA cell invasion and migration mediated by miR-339-5p was regulated by BCL6, miR-339-5p inhibitor and BCL6 siRNA were co-transfected into A549 cells. Then, RT-qPCR was carried out to examine the transfection efficiency. As presented in Fig. 4A, we found that the co-transfection of miR-339-5p inhibitor and BCL6 siRNA repressed the increased BCL6 expression induced by miR-339-5p inhibitor. Moreover, we performed western blot analysis to confirm the transfection efficiency. Similarly to the RT-qPCR results, the data revealed that BCL6 siRNA downregulated the BCL6 expression (Fig. 4B). Additionally, we investigated whether BCL6 downregulation was related to LA cell invasion and migration by performing Transwell assays. The results showed that knockout of BCL6 reversed the promoting functions of miR-339-5p inhibitor in LA cell invasion and migration (Fig. 4C and D). 


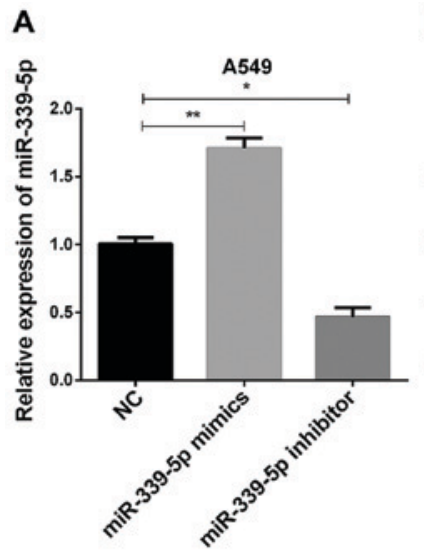

B

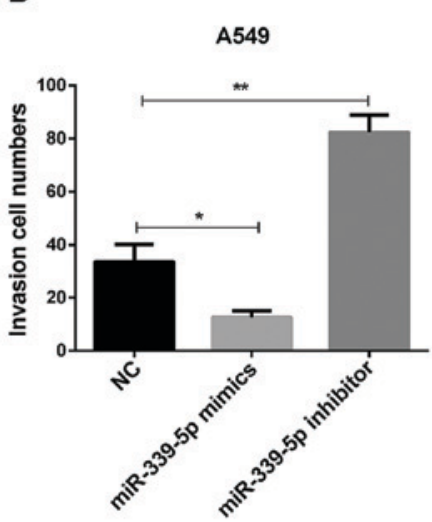

C

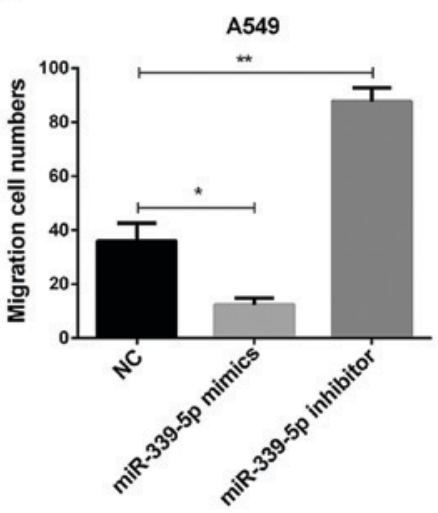

D

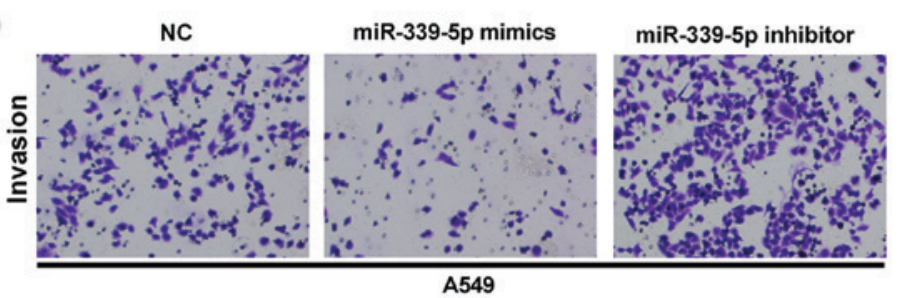

E

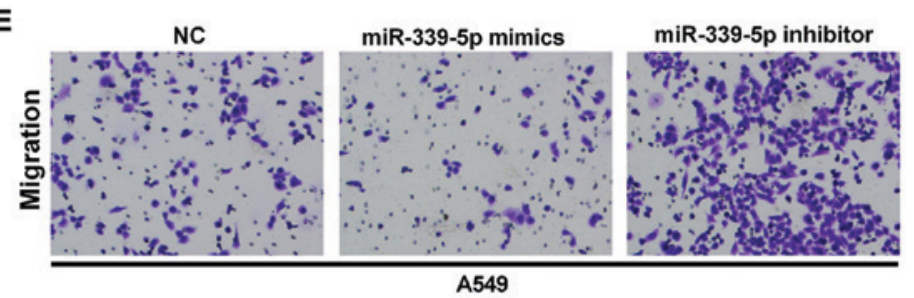

Figure 2. miR-339-5p overexpression suppresses invasion and migration of LA cells. (A) miR-339-5p expression in LA cells treated with miR-339-5p mimics or inhibitor were evaluated ("P $\left.<0.05,{ }^{* * *} \mathrm{P}<0.01\right)$. (B and $\left.\mathrm{C}\right)$ Quantitative results of Transwell assays in LA cells with different transfections $\left({ }^{*} \mathrm{P}<0.05,{ }^{* *} \mathrm{P}<0.01\right)$. (D and E) Invasion and migration capacities were observed by Transwell assays in LA cells with different transfections. LA, lung adenocarcinoma.

A

C

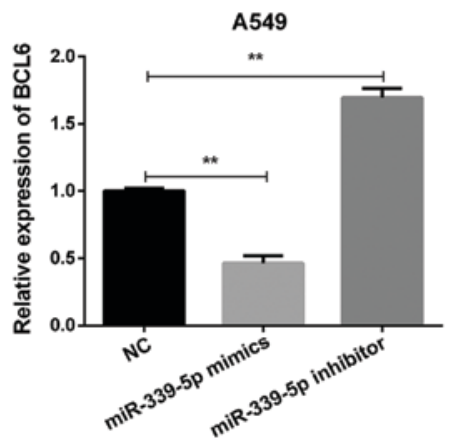

B

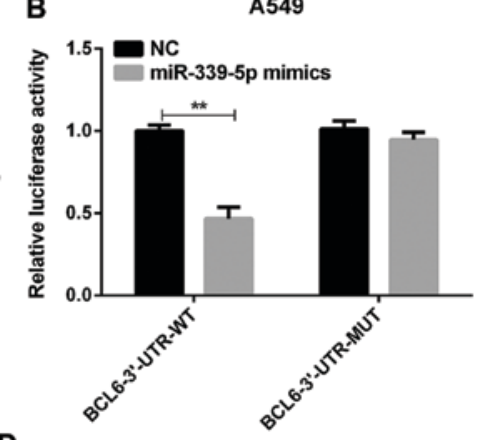

D

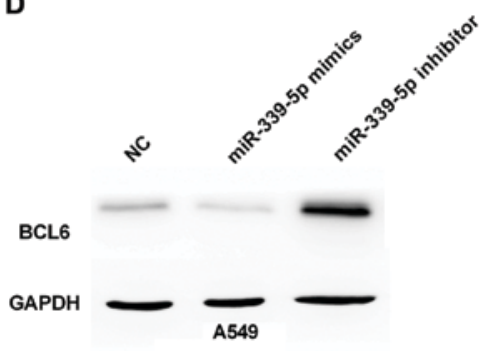

Figure 3. miR-339-5p modulates LA cells through targeting BCL6. (A) The BCL6 3'-UTRs containing the wild- or mutant-type miR-339-5p targeting sequence were inserted downstream of the luciferase reporter vector. (B) The Dual-Luciferase Reporter Assay revealed that the luciferase activities controlled by BCL6 3'-UTR were suppressed by ectopic miR-339-5p expression in LA cells ( $\left.{ }^{* *} \mathrm{P}<0.01\right)$. (C and D) RT-qPCR and western blot analysis were used to measure BCL6 mRNA and protein level in LA cell lines with transfection of miR-339-5p mimics or inhibitor $\left.{ }^{(* *} \mathrm{P}<0.01\right)$. LA, lung adenocarcinoma; BCL6, B-cell lymphoma 6; RT-qPCR, reverse transcription-quantitative PCR. 
A

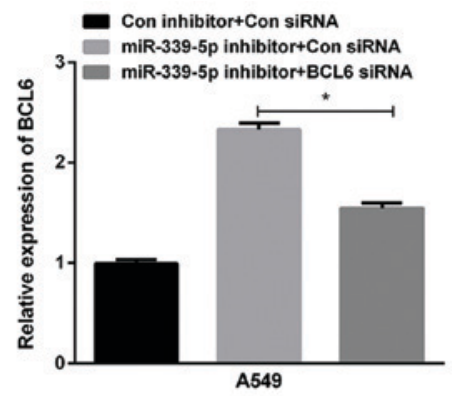

C

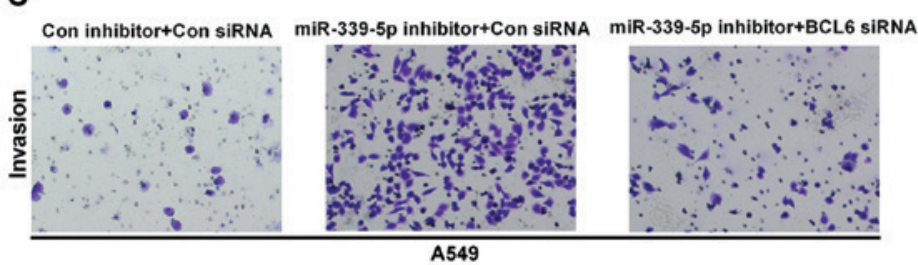

A549

B

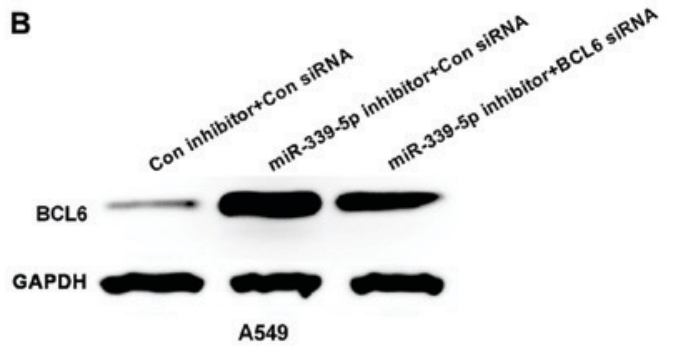

Con inhibitortCon siRNA

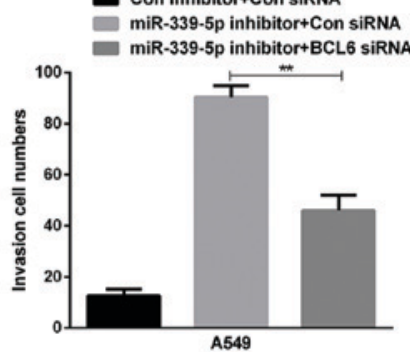

A549

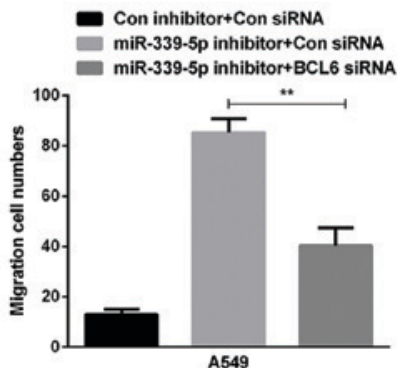

Figure 4. Knockdown of BCL6 abrogates the function mediated by miR-339-5p inhibitor in LA cell invasion and migration. (A and B) The BCL6 mRNA or protein expression level was measured by RT-qPCR or western blot analysis in LA cells co-transfected with BCL6 siRNA and miR-339-5p inhibitor ("P<0.05). (C and D) Transwell assays were conducted to detect invasion and migration ability in LA cells co-transfected with BCL6 siRNA and miR-339-5p inhibitor $\left({ }^{* *} \mathrm{P}<0.01\right)$. BCL6, B-cell lymphoma 6; LA, lung adenocarcinoma; RT-qPCR, reverse transcription-quantitative PCR.

\section{Discussion}

Lung cancer remains one of the major health problems for people worldwide due to its high incidence and mortality rates. LA is a common histologic type of lung tumors in various countries (19). Although many achievements have been made in antitumor therapies in recent years, the LA survival rate remains far from satisfactory. Owing to lack of specific clinical symptoms, LA patients are often diagnosed at late stages, having little chance to be effectively treated. Cancer-related molecular abnormality plays important roles in the therapies and progression of lung cancer (20). Growing evidence has demonstrated that the dysregulation of miRNAs is implicated in the LA process (21).

The abnormal miR-339-5p expression has been verified to play key roles in multiple tumors, including hepatocellular cancer (22) and breast cancer (23). For example, miR-339-5p was confirmed to regulate colorectal cancer metastasis, growth and colony formation via targeting PRL-1 (24). Jansson et al reported that miR-339-5p regulated the $\mathrm{p} 53$ tumor-suppressor pathway via regulating MDM2 (25). However, the clinical significance and potential molecular mechanism of miR-339-5p in LA are still unclear. The further evidence that miR-339-5p played key parts in human LA progression was provided in this study, given that miRNAs are comprehensively known as tumor regulators. Briefly, miR-339-5p was identified as the downregulated miRNA in LA by RT-qPCR. Besides, miR-339-5p overexpression was verified to inhibit the activities of LA cells by targeting BCL6. In short, the findings of this research revealed that miR-339-5p had important effects on LA development.

BCL6, as an oncogene in different tumors, has drawn much attention. For instance, BCL6 promoted glioma and served as a therapeutic target (26). high Lewis y antigen and BCL6 expressions in epithelial ovarian cancer were associated with poor prognosis and high tumor burden (27). BCL6 was increased and downregulated by miR-544 in triple-negative breast cancer to suppress tumor growth (28). To our knowledge, there is no previous research reported on the association between BCL6 and miR-339-5p in LA. Our present results provide preliminary strong evidence that BCL6 is directly targeted by miR-339-5p and implicated in LA. The data also revealed that knockdown of BCL6 reversed the effects of miR-339-5p inhibitor on LA cell invasion and migration. These data suggested that miR-339-5p exerted tumor suppressive roles in LA via targeting BCL6.

Taken together, the decrease of miR-339-5p expression in LA might be a potential biomarker, leading to a higher BCL6 expression. In addition, miR-339-5p overexpression could efficiently inhibit the LA cell invasion and migration abilities through regulating BCL6 expression. This finding will help to further understand the function of miR-339-5p in LA, and may provide insights into developing novel therapies. 


\section{Acknowledgements}

Not applicable.

\section{Funding}

No funding was received.

\section{Availability of data and materials}

The datasets used and/or analyzed during the present study are available from the corresponding author on reasonable request.

\section{Authors' contributions}

PL contributed significantly to statistical analysis and manuscript preparation. HL performed the experiments. YL revised the manuscript critically and contributed in the interpretation of the experimental results. YW analyzed and interpreted the data. LZ conceived and designed the study. HW contributed to the conception of the study. All authors read and approved the final manuscript.

\section{Ethics approval and consent to participate}

The study was approved by the Ethics Committee of People's Hospital of Rizhao (Rizhao, China). Patients who participated in this investigation, signed an informed consent and had complete clinical data.

\section{Patient consent for publication}

Not applicable.

\section{Competing interests}

The authors declare that they have no competing interests.

\section{References}

1. Torre LA, Bray F, Siegel RL, Ferlay J, Lortet-Tieulent J and Jemal A: Global cancer statistics, 2012. CA Cancer J Clin 65: 87-108, 2015.

2. Ferlay J, Shin HR, Bray F, Forman D, Mathers C and Parkin DM: Estimates of worldwide burden of cancer in 2008: GLOBOCAN 2008. Int J Cancer 127: 2893-2917, 2010.

3. Nana-Sinkam SP and Powell CA: Molecular biology of lung cancer: Diagnosis and management of lung cancer, 3rd ed American College of Chest Physicians evidence-based clinical practice guidelines. Chest 143 (5 Suppl): e30S-e39S, 2013.

4. Bhargava A, Bunkar N, Aglawe A, Pandey KC, Tiwari R, Chaudhury K, Goryacheva IY and Mishra PK: Epigenetic biomarkers for risk assessment of particulate matter associated lung cancer. Curr Drug Targets 19: 1127-1147, 2018.

5. Peng Z, Pan L, Niu Z, Li W, Dang X, Wan L, Zhang R and Yang S: Identification of microRNAs as potential biomarkers for lung adenocarcinoma using integrating genomics analysis. Oncotarget 8: 64143-64156, 2017.

6. Calin GA and Croce CM: MicroRNA signatures in human cancers. Nat Rev Cancer 6: 857-866, 2006.

7. Nohata N, Hanazawa T, Kinoshita T, Okamoto Y and Seki N: MicroRNAs function as tumor suppressors or oncogenes: Aberrant expression of microRNAs in head and neck squamous cell carcinoma. Auris Nasus Larynx 40: 143-149, 2013.
8. Yang L, Liang H, Wang Y, Gao S, Yin K, Liu Z, Zheng X, Lv Y, Wang L, Zhang CY, et al: MiRNA-203 suppresses tumor cell proliferation, migration and invasion by targeting Slug in gastric cancer. Protein Cell 7: 383-387, 2016.

9. Feng C, Sun P, Hu J, Feng H, Li M, Liu G, Pan Y, Feng Y, Xu Y, Feng K, et al: miRNA-556-3p promotes human bladder cancer proliferation, migration and invasion by negatively regulating DAB2IP expression. Int J Oncol 50: 2101-2112, 2017.

10. Hu X, Zhang M, Miao J, Wang X and Huang C: miRNA-4317 suppresses human gastric cancer cell proliferation by targeting ZNF322. Cell Biol Int: Sep 7, 2017 (Epub ahead of print).

11. Li Y, Zhang H, Dong Y, Fan Y, Li Y, Zhao C, Wang C, Liu J, Li X, Dong M, et al: MiR-146b-5p functions as a suppressor miRNA and prognosis predictor in non-small cell lung cancer. J Cancer 8: 1704-1716, 2017.

12. Wang D, Cao Q, Qu M, Xiao Z,Zhang M and Di S: MicroRNA-616 promotes the growth and metastasis of non-small cell lung cancer by targeting SOX7. Oncol Rep 38: 2078-2086, 2017.

13. Green MR, Vicente-Dueñas C, Alizadeh AA and Sánchez-García I: Hit-and-run lymphomagenesis by the Bcl6 oncogene. Cell Cycle 13: 1831-1832, 2014.

14. Kojima S, Hatano M, Okada S, Fukuda T, Toyama Y, Yuasa S, Ito $\mathrm{H}$ and Tokuhisa $\mathrm{T}$ : Testicular germ cell apoptosis in Bcl6-deficient mice. Development 128: 57-65, 2001.

15. Nola S, Sin S, Bonin F, Lidereau R and Driouch K: A methodological approach to unravel organ-specific breast cancer metastasis. J Mammary Gland Biol Neoplasia 17: 135-145, 2012.

16. Yan $\mathrm{H}$, Zhao M, Huang S, Chen P, Wu WY, Huang J, Wu ZS and $\mathrm{Wu}$ Q: Prolactin inhibits BCL6 expression in breast cancer cells through a microRNA-339-5p-dependent pathway. J Breast Cancer 19: 26-33, 2016

17. Kamada Y, Sakai N, Sogabe S, Ida K, Oki H, Sakamoto K, Lane W, Snell G, Iida M, Imaeda Y, et al: Discovery of a B-cell lymphoma 6 protein-protein interaction inhibitor by a biophysics-driven fragment-based approach. J Med Chem 60: 4358-4368, 2017.

18. Livak KJ and Schmittgen TD: Analysis of relative gene expression data using real-time quantitative PCR and the 2(-Delta Delta C(T)) method. Methods 25: 402-408, 2001.

19. Jemal A, Bray F, Center MM, Ferlay J, Ward E and Forman D: Global cancer statistics. CA Cancer J Clin 61: 69-90, 2011.

20. Rivera MP: Lung cancer in women: Differences in epidemiology, biology, histology, and treatment outcomes. Semin Respir Crit Care Med 34: 792-801, 2013

21. Wang Y, Han R, Chen Z, Fu M, Kang J, Li K, Li L, Chen H and He Y: A transcriptional miRNA-gene network associated with lung adenocarcinoma metastasis based on the TCGA database. Oncol Rep 35: 2257-2269, 2016.

22. Wang YL, Chen CM, Wang XM and Wang L: Effects of miR-339-5p on invasion and prognosis of hepatocellular carcinoma. Clin Res Hepatol Gastroenterol 40: 51-56, 2016.

23. Wu ZS, Wu Q, Wang CQ, Wang XN, Wang Y, Zhao JJ, Mao SS, Zhang GH, Zhang N and Xu XC: MiR-339-5p inhibits breast cancer cell migration and invasion in vitro and may be a potential biomarker for breast cancer prognosis. BMC Cancer 10: 542, 2010.

24. Zhou C, Liu G, Wang L, Lu Y, Yuan L, Zheng L, Chen F, Peng F and $\mathrm{Li} \mathrm{X}$ : MiR-339-5p regulates the growth, colony formation and metastasis of colorectal cancer cells by targeting PRL-1. PLoS One 8: e63142, 2013.

25. Jansson MD, Damas ND, Lees M, Jacobsen A and Lund AH: miR-339-5p regulates the p53 tumor-suppressor pathway by targeting MDM2. Oncogene 34: 1908-1918, 2015.

26. Xu L, Chen Y, Dutra-Clarke M, Mayakonda A, Hazawa M, Savinoff SE, Doan N, Said JW, Yong WH, Watkins A, et al: BCL6 promotes glioma and serves as a therapeutic target. Proc Natl Acad Sci USA 114: 3981-3986, 2017.

27. Zhu L, Feng H, Jin S, Tan M, Gao S, Zhuang H, Hu Z, Wang H, Song $\mathrm{Z}$ and Lin B: High expressions of BCL6 and Lewis y antigen are correlated with high tumor burden and poor prognosis in epithelial ovarian cancer. Tumour Biol 39: 1010428317711655, 2017, doi: $10.1177 / 1010428317711655$.

28. Zhu Z, Wang S, Zhu J, Yang Q, Dong $H$ and Huang J: MicroRNA-544 down-regulates both Bcl6 and Stat3 to inhibit tumor growth of human triple negative breast cancer. Biol Chem 397: 1087-1095, 2016. International (CC BY-NC-ND 4.0) License. 